\title{
Molecular and Crystal Structures of Two Chelating Agents for Use in MRI Contrast Agents
}

\author{
Lisbeth Ehnebom $^{\mathrm{a}, *}$ Berit Fjærtoft Pedersen ${ }^{\mathrm{a}}$ and Jo Klaveness ${ }^{\mathrm{a}, \mathrm{b}}$ \\ anstitute of Pharmacy, University of Oslo, PO Box 1068, Blindern, N-0316 Oslo, Norway and ${ }^{\mathrm{b}}$ Nycomed Imaging AS, \\ PO Box 4220, Torshov, N-0401 Oslo, Norway
}

\begin{abstract}
Ehnebom, L., Pedersen, B. F. and Klaveness, J., 1993. Molecular and Crystal Structures of Two Chelating Agents for Use in MRI Contrast Agents. - Acta Chem. Scand. 47: 965-970.

1,7-Bis-[( $N$-benzylcarbamoyl $)$ methyl $]-1,4,7$-triazaheptane-1,4,7-triacetic acid dihydrate, DTPA-BBA, $\mathrm{C}_{28} \mathrm{H}_{41} \mathrm{~N}_{5} \mathrm{O}_{10}$, crystallizes in the monoclinic space group $P 2 / a, Z=4$ with unit cell dimensions $a=18.019(5), b=7.204(1), c=24.288(7) \AA$ $\beta=99.26(2)^{\circ} . \quad R=0.034$ for 1916 unique reflections. 1,7-Bis- $[(N$-methylcarbamoyl)methyl ]-1,4,7-triazaheptane-1,4,7-triacetic acid dihydrate, DTPABMA, $\mathrm{C}_{16} \mathrm{H}_{33} \mathrm{~N}_{5} \mathrm{O}_{10}$, crystallizes in the monoclinic space group $P 2_{1} / c, Z=4$ with unit cell dimensions $a=17.472(4), b=7.110(2), c=17.846(2) \AA, \beta=96.02(1)^{\circ}$. $R=0.043$ for 4513 unique reflections.

There are two intramolecular hydrogen bonds in both molecules, which give them a folded conformation. The molecules crystallize as zwitterions with the central of the three amine nitrogen atoms (N3) protonated. An extensive hydrogenbonding network is found for both structures, including a short unsymmetrical carboxyl-carboxylate hydrogen bond, 2.522(3) $\AA$ (DTPA-BBA) and 2.504(2) $\AA$ (DTPA-BMA), respectively, and an almost equally short carboxyl-water hydrogen bond, $2.570(4) \AA$ (DTPA-BBA) and 2.575(2) $\AA$ (DTPA-BMA), respectively.
\end{abstract}

Various paramagnetic contrast agents for magnetic resonance imaging (MRI) are now available and in clinical development. These paramagnetic compounds are metal chelates based on manganese $\left(\mathrm{Mn}^{2+}\right)$, gadolinium $\left(\mathrm{Gd}^{3+}\right)$ or dysprosium $\left(\mathrm{Dy}^{3+}\right)$. The efficacy, toxicity; biodistribution and bioelimination of the chelates are dependent on physicochemical parameters such as molecular weight, charge, metal ion selectivity, HLB (hydrophilic-lipophilic balance) and the overall chemical structure of the chelate. ${ }^{1}$ In general, the chelating agent should have a high selectivity for the paramagnetic ion to secure an in vivo stable chelate, and neutral, hydrophilic chelates are less toxic than ionic chelates with hydrophobic groups. The latter compounds usually have a higher liver uptake than pure hydrophilic agents.

The compounds studied here are two bis amides of DTPA; DTPA-bis-(methylamide) (DTPA-BMA) and DTPA-bis-(benzylamide) (DTPA-BBA). DTPA-BMA is the chelating agent in two paramagnetic MRI contrast agents based on gadolinium (Omniscan $\left.{ }^{\circledR}, \mathrm{S}-041\right)^{2}$ and dysprosium (S-043).,4 DTPA-BBA is a more hydrophobic chelating agent for gadolinium and dysprosium. Investigations of lanthanide DTPA-BBA chelates are in progress.

* To whom correspondence should be addressed.<smiles>O=C(O)CN1CCN(C(=O)C=NCc2ccccc2)CC[NH+](CC(=O)O)CN(CC(=O)O)C1</smiles>

DTPA-BBA<smiles>CNC(=O)CN1CC[NH+](CC(=O)O)CC[NH+](CC(=O)O)C1</smiles>

DTPA-BMA

\section{Experimental}

Crystals suitable for X-ray investigation were obtained by slow evaporation from a methanol-ethanol-water solution (DTPA-BBA). For DTPA-BMA they were made by vapour diffusion from a water-methanol system. In both instances the crystals were colourless and needle-shaped.

Data collections were performed on a Nicolet $P 3 / F$ automatic diffractometer with the $\theta-2 \theta$ scan technique. For DTPA-BBA the test reflections were used to normalize the collected material due to some fluctuation. The settings of 25 general reflections were used in a least-squares fit to determine the unit cell parameters, 
Table 1. Crystal data and intensity collection.

\begin{tabular}{|c|c|c|}
\hline & DTPA-BBA & DTPA-BMA \\
\hline $\begin{array}{l}\text { Formula } \\
\text { Formula weight } / \mathrm{g} \mathrm{mol}^{-1} \\
\text { Crystal dimensions } / \mathrm{mm}^{3} \\
\text { Density calculated } / \mathrm{Mg} \mathrm{m}^{-3} \\
\text { Linear absorption coefficient, } \mu / \mathrm{cm}^{-1} \\
F(000) \\
\text { Space group } \\
Z \\
a / \AA \\
b / \AA \\
c / \AA \\
\beta / \AA \\
V / \AA^{3} \\
\text { Diffractometer } \\
\text { Radiation } \\
\text { Wavelength } / \AA \\
\text { Monochromator } \\
\text { Temperature } / \mathrm{K} \\
\text { Scan mode } \\
\text { Scan range } /^{\circ} \\
\text { Scan speed } /{ }^{\circ} \text { min } \\
2 \theta \text { range } /^{\circ} \\
\text { Background } / \text { scan ratio } \\
\text { No. of reflections measured } \\
\text { No. of unique reflections }[/>3 \sigma(/)] \\
\text { Stability monitoring } \\
R=\Sigma\left(\left|F_{\mathrm{o}}\right|-\left|F_{\mathrm{c}}\right|\right) / \Sigma\left|F_{\mathrm{o}}\right| \\
R \mathrm{w}=\left[\sum w\left(\left|F_{\mathrm{o}}\right|-\left|F_{\mathrm{c}}\right|\right)^{2} / \Sigma w\left|F_{\mathrm{o}}\right|^{2}\right]^{1 / 2} \\
S=\left(\sum w \Delta^{2}\right) / n-p\end{array}$ & $\begin{array}{l}\mathrm{C}_{28} \mathrm{H}_{41} \mathrm{~N}_{5} \mathrm{O}_{10} \\
607.66 \\
0.35 \times 0.3 \times 0.1 \\
1.29 \\
0.93 \\
1296 \\
P 2{ }_{1} / \mathrm{a}(\mathrm{No} .14) \\
4 \\
18.019(5) \\
7.204(1) \\
24.288(7) \\
99.26(2) \\
3112(1) \\
\text { Nicolet } P 3 / F \\
\text { MoKa } \\
0.71069 \\
\mathrm{Graphite} \\
295 \\
\theta-2 \theta \\
2 \theta \mathrm{a}_{1}-0.8 \text { to } 2 \theta \mathrm{a}_{2}+1.0 \\
3-6 \\
3.0-40.0 \\
0.7 \\
3262 \\
1916 \\
3 \text { test refl. } / 135 \text { observ. } \\
0.034 \\
0.031 \\
1.61\end{array}$ & $\begin{array}{l}\mathrm{C}_{16} \mathrm{H}_{33} \mathrm{~N}_{5} \mathrm{O}_{10} \\
455.46 \\
1.9 \times 0.3 \times 0.1 \\
1.37 \\
1.07 \\
976 \\
P 2_{1} / c(\text { No. } 14) \\
4 \\
17.472(4) \\
7.110(2) \\
17.846(2) \\
96.02(1) \\
2204.7(6) \\
\text { Nicolet } P 3 / F \\
\text { MoKa } \\
0.71069 \\
\text { Graphite } \\
138 \\
\theta-2 \theta \\
2 \theta a_{1}-1.0 \text { to } 2 \theta a_{2}+1.2 \\
3 \\
3.0-60.0 \\
0.7 \\
7210 \\
4513 \\
2 \text { test refl. } 135 \text { observ. } \\
0.043 \\
0.043 \\
2.32\end{array}$ \\
\hline
\end{tabular}

$14<2 \theta<26^{\circ}$ (DTPA-BBA) and $25<2 \theta<40^{\circ}$ (DTPABMA), respectively. The intensity data have been corrected for Lorentz and polarization effects, but no absorption correction has been applied. Further details concerning crystallographic data and experimental conditions are summarized in Table 1.

\section{Structure determination and refinement}

$D T P A-B B A$. The structure was solved by direct methods (MITHRIL), ${ }^{5}$ which gave 31 out of 43 non-hydrogen atoms. Based on these atoms a Fourier synthesis was calculated, ${ }^{6}$ and all the other non-hydrogen atoms, except one of the aromatic carbon atoms (C4) and the water oxygen atoms, were located. After some cycles of leastsquares refinement, ${ }^{6}$ the missing non-hydrogen atoms were located from difference Fourier syntheses. Alternating least-squares refinement and difference Fourier syntheses gave 26 out of 41 hydrogen atoms prior to inclusion of anisotropic vibration for the non-hydrogen atoms.

The local version of the full-matrix least-squares refinement program used so far allows only 350 parameters to be refined simultaneously. A block-diagonal matrix program was used alternating with difference Fourier syntheses while locating the remaining hydrogen atoms. The hydrogen atoms attached to aromatic carbon atoms were included at positions calculated from known geometry.
In the final stages of refinement the full-matrix program was reintroduced. The structure was first refined without, and then with, only the aromatic rings. In the next step all the atomic coordinates, but no thermal parameters, were refined. Then repeated cycles with one of the aromatic rings included at a time were performed.

DTPA-BMA. Phases were obtained using direct methods (MITHRIL). All non-hydrogen atoms, including water oxygen atoms, were located from a Fourier synthesis based on $F_{\text {obs }}$. The parameters for these atoms were then refined by least-squares refinement.

After anisotropic vibration had been included and the structure refined, all the hydrogen atoms were found from difference Fourier syntheses. As stated above, the fullmatrix program allows only 350 parameters to be refined simultaneously. For this reason the structure was refined without the water molecules when the atomic coordinates of hydrogen were included. The parameters of the water molecules were then refined separately.

For both structures the hydrogen atoms were given a $B$-value $0.2 \AA^{2}$ larger than that of the atoms to which they are attached. All least-squares refinement was carried out by minimization of $\Sigma w(\Delta F)^{2}$, where $w=1 / \sigma^{2}$. The final difference map gave maximum and minimum electron densities of \pm 0.15 e $\AA^{-3}$ (DTPA-BBA) and \pm 0.40 e $\AA^{-3}$ (DTPA-BMA), respectively. Atomic scattering factors were taken from Ref. 7. 
Table 2. Final fractional atomic coordinates with e.s.d.s in parentheses and equivalent isotropic temperature factors (in $\AA^{2}$ ).

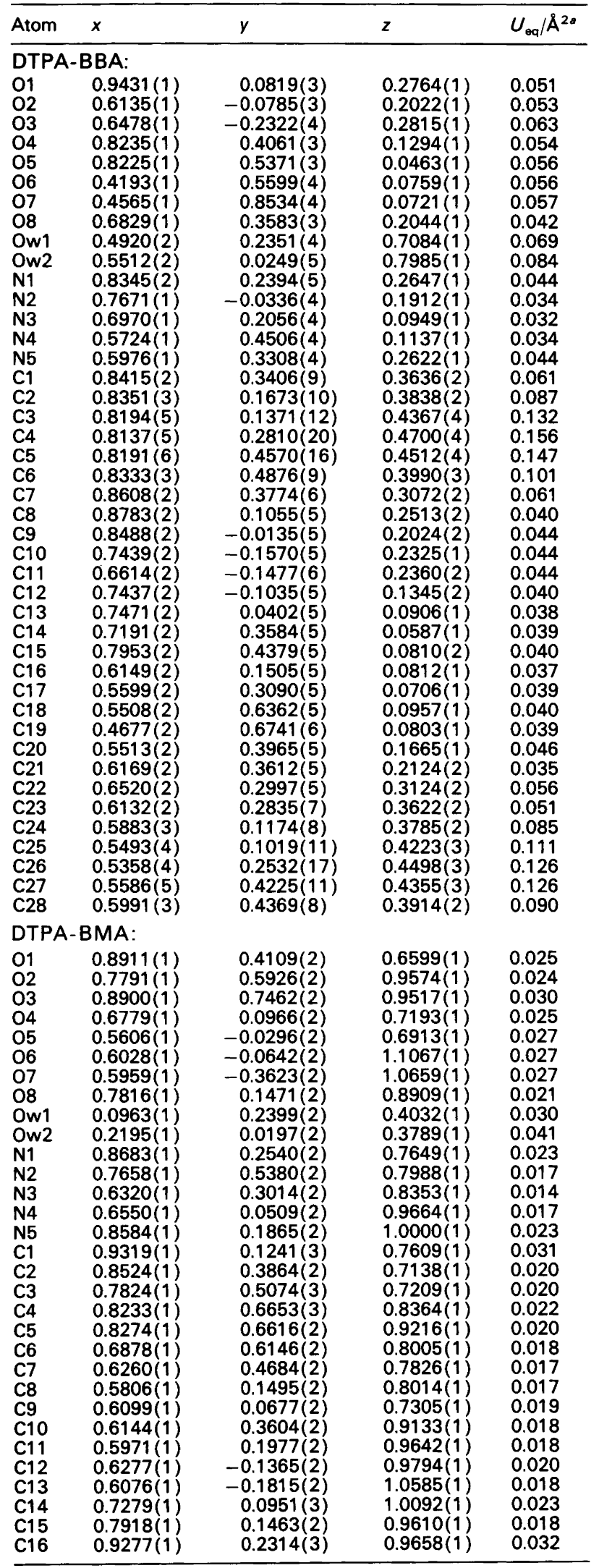

${ }^{a} U_{\mathrm{eq}}=\frac{1}{3} \Sigma_{i} \Sigma_{j} \cdot U_{i j} \cdot a_{i}^{*} \cdot a_{j}{ }^{*} \cdot a_{i} \cdot a_{j}$.
Table 3. Interatomic distances (in $\AA$ ), with e.s.d.s in parentheses.

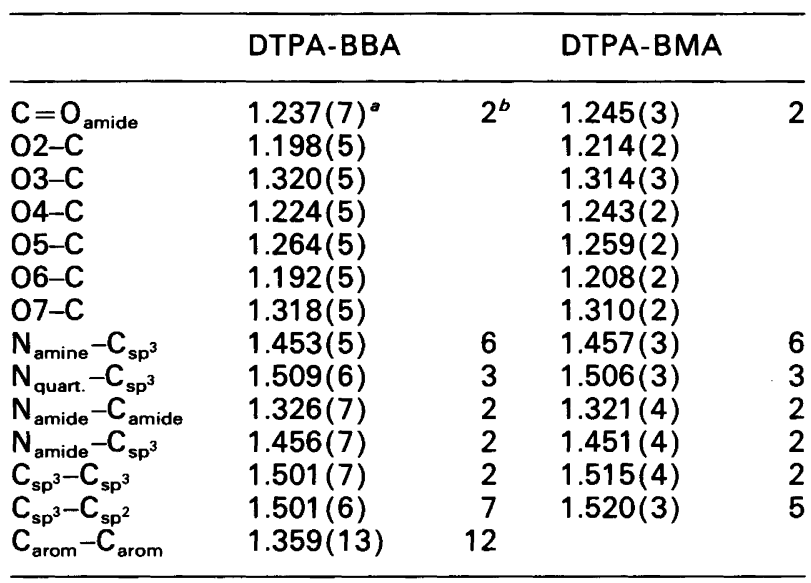

${ }^{a}$ Mean value. ${ }^{b}$ No. of observations.

\section{Results and discussion}

Final fractional coordinates and equivalent temperature factors are given in Table 2.

Geometry. Bond lengths are given in Table 3. As found for the dysprosium complex with DTPA-BMA, ${ }^{3}$ the bonds between $\mathrm{sp}^{3}$ carbon atoms are somewhat short. This is in agreement with results reported from structural work on $\alpha$ - and $\beta$-EDTA. ${ }^{8-10}$ One notes that the average $\mathrm{C}_{\text {arom }}-\mathrm{C}_{\text {arom }}$ bond length of $1.359(13) \AA$ is rather short. However, this should be seen in relation to the rather pronounced thermal motion of the phenyl goups, $c f$. Table 2 and Fig. 1.

The carbon-oxygen bond lengths in carboxylate groups should be almost equal. It is, however, seen from Table 3 that the carbon ... O5 bonds are longer than the

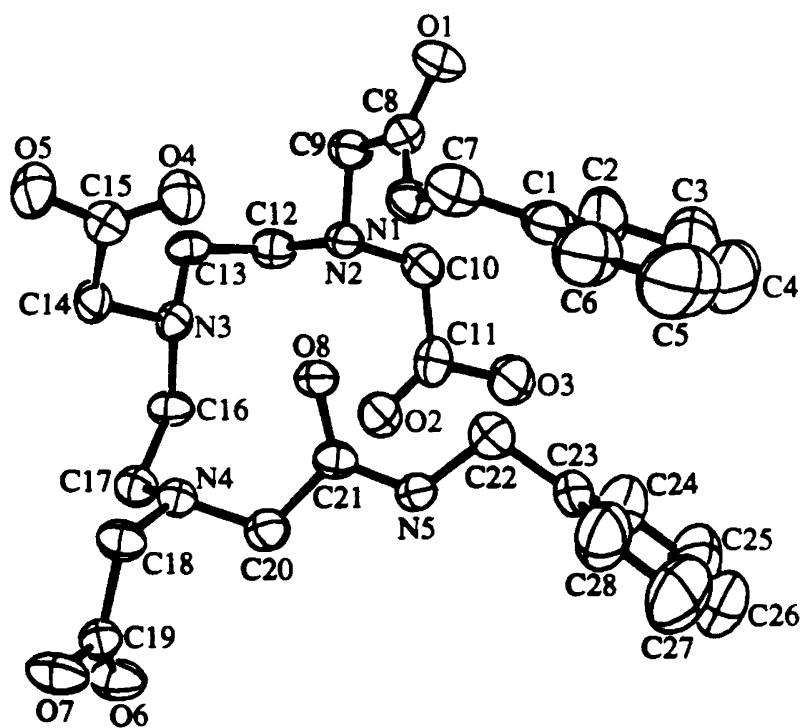

Fig. 1. Perspective drawing of DTPA-BBA with thermal ellipsoids of vibration ( $50 \%$ probability); hydrogen atoms have been omitted for clarity. 
Table 4. Intra- and intermolecular hydrogen bonds, bond lengths (in $\AA$ ) and angles (in ${ }^{\circ}$ ) with e.s.d.s in parentheses.

\begin{tabular}{|c|c|c|c|c|c|c|}
\hline & \multicolumn{3}{|c|}{ DTPA-BBA } & \multicolumn{3}{|c|}{ DTPA-BMA } \\
\hline & $D^{a} \cdots A^{b}$ & $\mathrm{H}-\mathrm{A}$ & $\mathrm{D}-\mathrm{H} \cdots \mathrm{A}$ & $D \cdots A$ & $\mathrm{H}-\mathrm{A}$ & $D-H \cdots A$ \\
\hline $\begin{array}{l}\text { N1-H711 } \cdots \text { O } \\
\text { N3-H731 } \cdots \text { O8 } \\
\text { O7-H871 } \cdots \text { O5 } \\
\text { O3-H831 } \cdots \text { Ow1 } \\
\text { Ow1-Hw12 } \cdots \text { O1 } \\
\text { Ow2-Hw22 } \cdots \text { O } 2 \\
\text { Ow2-Hw21 } \cdots \text { O4 } \\
\text { Ow1-Hw11 } \cdots \text { Ow2 } \\
\text { N5-H751 } \cdots \text { O } 1\end{array}$ & $\begin{array}{l}3.010(4) \\
2.928(3) \\
2.522(3) \\
2.570(4) \\
2.758(4) \\
2.992(4) \\
2.762(4) \\
2.734(5) \\
2.928(3)\end{array}$ & $\begin{array}{l}2.11(3) \\
2.06(3) \\
1.63(3) \\
1.62(3) \\
1.95(4) \\
2.29(4) \\
1.88(4) \\
1.78(3) \\
2.11(3)\end{array}$ & $\begin{array}{l}164(3) \\
162(3) \\
176(3) \\
174(3) \\
174(4) \\
142(3) \\
177(3) \\
168(3) \\
163(3)\end{array}$ & $\begin{array}{l}2.940(2) \\
2.912(2) \\
2.504(2) \\
2.575(2) \\
2.745(2) \\
2.965(2) \\
2.761(2) \\
2.734(2) \\
2.933(2)\end{array}$ & $\begin{array}{l}2.13(2) \\
2.06(2) \\
1.61(2) \\
1.69(2) \\
1.87(2) \\
2.30(2) \\
1.86(2) \\
1.87(2) \\
2.13(2)\end{array}$ & $\begin{array}{l}167(2) \\
159(2) \\
175(2) \\
171(2) \\
175(2) \\
142(2) \\
169(2) \\
163(2) \\
165(2)\end{array}$ \\
\hline
\end{tabular}

${ }^{a}$ Donor. ${ }^{b}$ Acceptor.

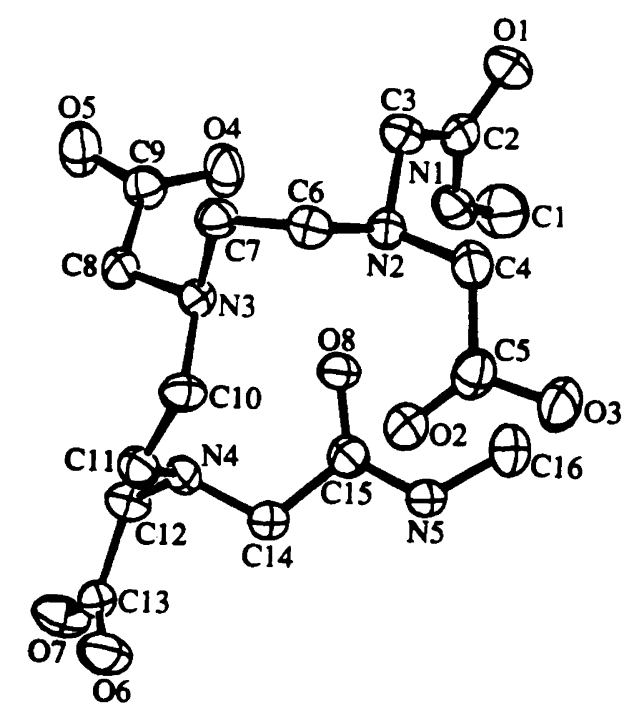

Fig. 2. Perspective drawing of DTPA-BMA with thermal ellipsoids of vibration ( $90 \%$ probability); hydrogen atoms have been omitted for clarity.

carbon... O4. This is likely to be a result of the $\mathrm{O} 5$ atom participating in a short hydrogen bond in both molecules as discussed below.

Conformation and intramolecular hydrogen bonding. The molecules with atomic numbering schemes are shown in Figs. 1 and 2 (ORTEP) ${ }^{6}$ It can be seen that their conformations are rather similar. The structures are stabilized by two intramolecular hydrogen bonds in corresponding positions. The hydrogen donors are the protonated amine nitrogen atom (N3) and one of the amide nitrogen atoms (N1), and the amide oxygen (O8) at the opposite end of the molecule is the acceptor. The lengths of the intramolecular hydrogen bonds in DTPA-BBA are $\mathrm{O} 8 \cdots \mathrm{N} 1=3.010(4) \AA$ and $\mathrm{O} 8 \cdots \mathrm{N} 3=2.928(3) \AA$, with donor-H $\cdots$ acceptor angles of $164(3)^{\circ}$ and $162(3)^{\circ}$, respectively. The corresponding values for DTPA-BMA are $2.940(2) \AA / 167(2)^{\circ}$ and $2.912(2) \AA / 159(2)^{\circ}$. The geometry of the intra- and intermolecular hydrogen bonds is presented in Table 4.
The hydrogen bonding results in a folded conformation, resembling the conformation the molecules adopt as ligands in chelates with lanthanide ions. Because the compounds already exist in a favourable conformation there is reason to believe that the energy needed to form chelates is less than it would have been from an extended form. Formation of chelates is, however, often impaired by intramolecular hydrogen bonds, because of the energy needed to disrupt them. The intramolecular hydrogen bonds have the opposite effect on chelation if they decrease the desolvation energy of free ligands. ${ }^{11}$

The torsional angles of the main-chain (regarding the acetic acid groups and the aromatic rings as substituents) are given in Table 5. In both molecules there is a preference for gauche bonds over anti, which is probably a result of the two intramolecular hydrogen bonds. The two molecules have gauche and anti bonds of almost equal values in corresponding positions, the only difference being that their signs are opposite. The difference in sign is merely a result of the chosen sign of the atomic coordinates for the two molecules, since both are centrosymmetric.

Zwitterions. Both molecules exist as zwitterions with the central of the three amine nitrogen atoms (N3) protonated. The hydrogen atom of the protonated amine

Table 5. Torsional angles of the main-chain in the two molecules (in ${ }^{\circ}$ ), with e.s.d.s in parentheses.

\begin{tabular}{lrlr}
\hline DTPA-BBA & \multicolumn{3}{l}{ DTPA-BMA } \\
\hline C7-N1-C8-C9 & $-172.5(6)$ & C1-N1-C2-C3 & $176.8(3)$ \\
N1-C8-C9-N2 & $-28.5(4)$ & N1-C2-C3-N2 & $31.8(2)$ \\
C12-N2-C9-C8 & $163.1(4)$ & C6-N2-C3-C2 & $-165.8(2)$ \\
C9-N2-C12-C13 & $-74.9(4)$ & C3-N2-C6-C7 & $74.7(2)$ \\
N2-C12-C13-N3 & $-60.7(3)$ & N2-C6-C7-N3 & $58.6(2)$ \\
C16-N3-C13-C12 & $-68.2(3)$ & C10-N3-C7-C6 & $68.2(2)$ \\
C13-N3-C16-C17 & $-165.9(4)$ & C7-N3-C10-C11 & $163.9(2)$ \\
N3-C16-C17-N4 & $-49.5(3)$ & N3-C10-C11-N4 & $50.3(2)$ \\
C20-N4-C17-C16 & $-72.1(4)$ & C14-N4-C11-C10 & $73.8(2)$ \\
C17-N4-C20-C21 & $107.9(4)$ & C11-N4-C14-C15 & $-103.1(2)$ \\
N4-C20-C21-N5 & $173.3(5)$ & N4-C14-C15-N5 & $-179.9(3)$ \\
C22-N5-C21-C20 & $-178.0(5)$ & C16-N5-C15-C14 & $177.4(3)$ \\
\hline
\end{tabular}



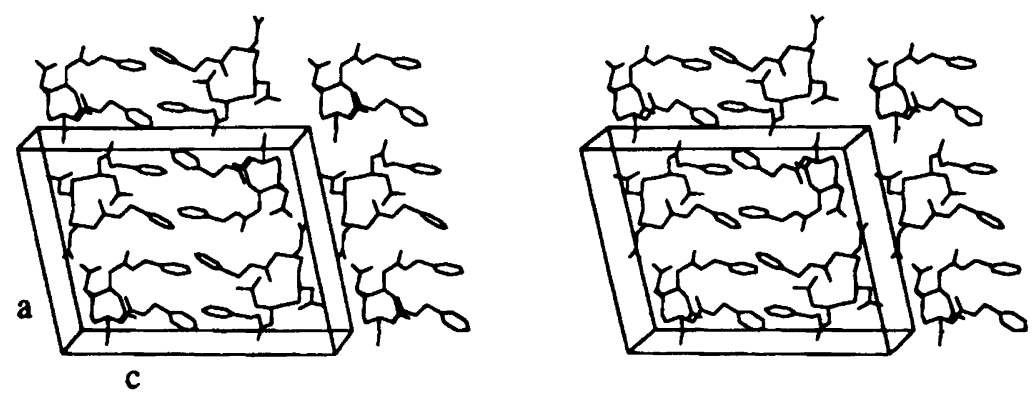

Fig. 3. Stereoscopic view showing the packing pattern of DTPA-BBA.

nitrogen and the ones attached to the two carboxyl groups were located from difference Fourier syntheses. Bond lengths between amine nitrogen atoms (N2 and N4) and $\mathrm{sp}^{3}$ carbon atoms are $1.453(5) \AA$ (DTPA-BBA) and $1.457(3) \AA$ (DTPA-BMA), respectively. Bonds between the third amine nitrogen atom (N3) and $\mathrm{sp}^{3}$ carbon atoms are longer as a consequence of the protonation; $1.509(6) \AA$ for DTPA-BBA and 1.506(3) $\AA$ for DTPA$B M A$, respectively. Ref. 12 reports an average value of $1.502 \AA$ for this type of bond. Bond lengths (Table 3 ) and angles in the carboxylic acid groups support the presence of the zwitterions. The angles in the carboxyl groups of both molecules show the following average values; $\mathrm{O}=\mathrm{C}-\mathrm{C}=125.1(3)^{\circ}, \mathrm{O}=\mathrm{C}-\mathrm{O}=124.8(3)^{\circ}$ and $\mathrm{O}-\mathrm{C}-\mathrm{C}=110.3(4)^{\circ}$. The carboxylate groups have a mean $\mathrm{O} \cdots \mathrm{C} \cdots \mathrm{O}$ angle of $127.4(5)^{\circ}$, the $\mathrm{O} 4 \cdots \mathrm{C}-\mathrm{C}$ angle is $119.0(5)^{\circ}$ and the angle $\mathrm{O} 5 \cdots \mathrm{C}-\mathrm{C}$ is $113.7(5)^{\circ}$. The difference between the two $\mathrm{O} \cdots \mathrm{C}-\mathrm{C}$ angles in the carboxylate groups should be seen in relation to the longer carbon ... O5 bond, which is a consequence of the short intermolecular hydrogen bond.

Packing pattern, intermolecular hydrogen bonds. The arrangement of molecules in the unit cell is shown in Fig. 3 for DTPA-BBA and Fig. 4 for DTPA-BMA (PLUTO). ${ }^{13}$ The unit cell contains four molecules in both structures.

There is an extensive two-dimensional hydrogenbonding network in both structures. The hydrogen bonds connect the DTPA-BBA molecules along the $a$ - and $b$-axes, whilst for DTPA-BMA the hydrogen bonds are along the $b$ - and $c$-axes. DTPA-BBA has an axial glide plane along the $a$-axis, DTPA-BMA along the $c$-axis. The hydrogen-bonding network is very similar for the two structures (Table 4). The two water molecules of crystallization link the molecules in both structures together with hydrogen bonds and are also hydrogen-bonded to each other.

Both structures contain one strong hydrogen bond from $\mathrm{O} 5$ of the carboxylate group to $\mathrm{O} 7$ of a symmetry related carboxyl group. The lengths are $2.522(3) \AA$ in DTPA-BBA and 2.504(2) $\AA$ in DTPA-BMA, respectively. They are both unsymmetrical and linear, with the hydrogen atom located closest to 07 . After least-squares refinement of the positional parameters for the hydrogen atoms the geometries of the two short hydrogen bonds were the following: $\mathrm{O} 7-\mathrm{H} 871=0.89(4) \AA$, $\mathrm{H} 871 \cdots \mathrm{O} 5=$ 1.63(3) $\AA$, O7-H871 $\ldots \mathrm{O} 5=176(3)^{\circ}$ for DTPA-BBA, and $\mathrm{O} 7-\mathrm{H} 871=0.89(2) \AA, \quad \mathrm{H} 871 \cdots \mathrm{O} 5=1.61(2) \AA$, O7-H871 $\cdots$ O5 $=175(2)^{\circ}$ for DTPA-BMA. In $\beta$-EDTA there is a very short carboxyl-carboxylate hydrogen bond $(2.46 \AA)$ which is approximately symmetrical., ${ }^{8,9}$ In $\alpha$-EDTA it has a value of $2.47 \AA$ and is unsymmetrical. ${ }^{10}$ Hydrogen bonds in general, and very short hydrogen bonds in acid salts $(2.4-2.5 \AA)$ in particular, are discussed by Speakman. ${ }^{14}$ The molecules also have an almost equally short hydrogen bond between $\mathrm{O} 3$ of the other carboxyl group and the water molecule Ow1. Corre-
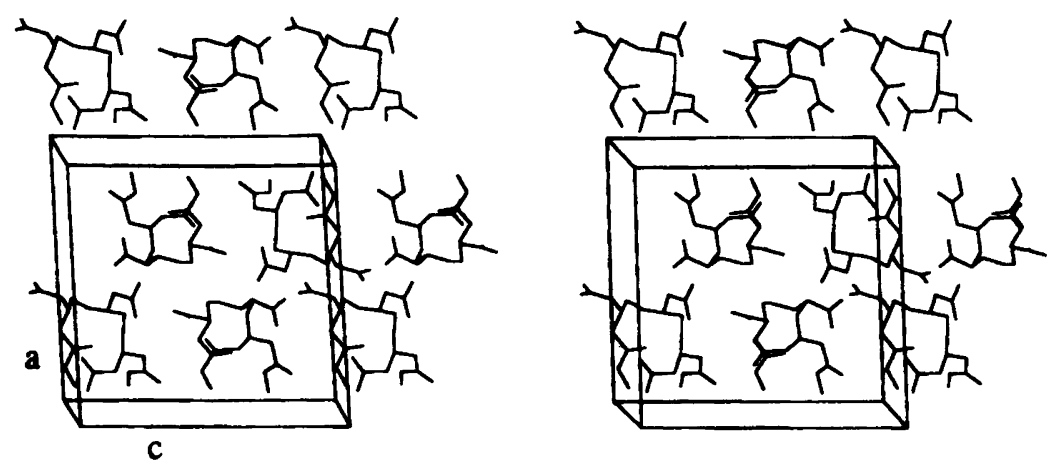

Fig. 4. Stereoscopic view showing the packing pattern of DTPA-BMA. 
sponding lengths are 2.570(4) $\AA$ (DTPA-BBA) and $2.575(2) \AA$ (DTPA-BMA), respectively.

It can be seen from Fig. 3 that the aromatic rings in DTPA-BBA are stacked in between each other. The average closest carbon-carbon separation between rings is $3.8 \AA$. This stacking along the $a$-axis may have a stabilizing effect on the crystal structure.

Acknowledgment. The authors thank members of the crystallographic group at the University of Oslo, Department of Chemistry, for fruitful discussions and for assistance during the data collection.

\section{References}

1. Watson, A. D., Rocklage, S. M. and Carvlin, M. J. In: Stark, D. D. and Bradley, W. G., Eds., Magnetic Resonance Imaging, 2nd edn., Vol. 1, Chap. 4, Mosby-Year Book Inc., St. Louis 1992.

2. Greco, A., McNamara, M. T., Lanthiez, P., Quay, S. C. and Michelozzi, G. Radiology 176 (1990) 451.
3. Ehnebom, L. and Pedersen, B. F. Acta Chem. Scand. 46 (1992) 126.

4. Saeed, M., Wendland, M. F., Takehara, I. and Higgins, C. B. Radiology 175 (1990) 633.

5. Gilmore, C. J. J. Appl. Crystallogr. 17 (1984) 42.

6. Mallinson, P. R. and Muir, K. W. J. Appl. Crystallogr. 18 (1985) 51 .

7. International Tables for X-Ray Crystallography, Vol. 4, Kynoch Press, Birmingham 1974.

8. Cotrait, M. Acta Crystallogr., Sect. B 28 (1972) 781.

9. Ladd, M. F. C. and Povey, D. C. J. Cryst. Mol. Struct. 3 (1973) 15

10. Ladd, M. F. C., Povey, D. C. and Stace, B. C. J. Cryst. Mol. Struct. 4 (1974) 313.

11. Fossheim, R., Dugstad, H. and Dahl, S. G. J. Med. Chem. 34 (1991) 819 .

12. International Tables for Crystallography, Vol. C, pp. 691706, Kluwer Academic Publishers, Dordrecht 1992.

13. Motherwell, W. D. S., PLUTO, University of Cambridge, Cambridge, UK 1976.

14. Speakman, J. C. In: Robertson, J. M., Ed., Chemical Crystallography, Vol. 11, Chap. 1, Butterworths, London 1972.

Received April 4, 1993. 